\title{
One-particle inclusive distribution in the unitarized pomeron models
}

\author{
A. Alkin ${ }^{1}$, E. Martynov ${ }^{1}$, O. Romanetc ${ }^{2}$, V. Pauk ${ }^{* 2}$
}

${ }^{1}$ Bogolyubov Institute for Theoretical Physics

Metrologichna 14b, Kiev, UA-03680, Ukraine

${ }^{2}$ National Taras Shevchenko University of Kiev

Volodimirska 60, Kiev, UA-03101, Ukraine

E-mail: martynovebitp.kiev.ua, paukvpegmail.com

\begin{abstract}
High energy inclusive hadron production in the central kinematical region is considered within the models of unitarized pomeron. It is shown that starting from the pomeron contribution with intercept $\alpha_{P}(0)>1$ one can obtain in a quasieikonal approach inclusive cross section which is similar to contribution of triple pole (at $t=0$ ) pomeron. Basing on this analogy we then consider general form of tripole and dipole pomeron contribution to inclusive cross section. They lead to a parabolic form of the distribution in rapidity giving $<n>\propto \ln ^{3} s$ (tripole) or $<n>\propto \ln ^{2} s$ (dipole). With suggested parametrization of $p_{t}$ dependence of cross sections the considered models well describe the data for charged hadron distributions in $p p$ and $\bar{p} p$ interactions at energy $\sqrt{s} \geq 200$ $\mathrm{GeV}$. Predictions for LHC energy as well as comparison with a simulation of one particle inclusive production are given.
\end{abstract}

XXth Hadron Collider Physics Symposium

November 16 - 20, 2009

Evian, France

* Speaker. 
The model of pomeron with intercept $\alpha_{P}(0)=1+\varepsilon, \varepsilon>0$ is very attractive from the phenomenological point of view [1]. Firstly it gives a simple and compact parametrization for many high-energy soft processes (elastic and deep inelastic scattering, diffraction and others). Secondly it describes quite well a lot of data for high enough energy (for example, total cross sections and small- $t$ elastic scattering at $\sqrt{s} \geq 5 \mathrm{GeV}$ ).

However the contribution of supercritical pomeron to the total cross-section rises with energy as a power $\sigma \propto s^{\Delta}$, violating the Froissart-Martin bound $\sigma_{t o t}<$ const $\ln ^{2}\left(s / s_{0}\right)$. The strict consistent procedure of unitarization is absent now, but there are some simple phenomenological ways to eliminate the rough contradictions with the unitarity. The most simple method to do that is summing multipomeron diagrams. If $\alpha_{P}(t)=1+\varepsilon+\alpha_{P}^{\prime} t$ and hadron-pomeron coupling $g_{a, b}(t)=\exp \left(B_{a, b} t\right)$ one can find that at $s \rightarrow \infty$ in this model $\sigma_{\text {tot }}(s) \approx 8 \pi \varepsilon R^{2}(s) \ln \left(s / s_{0}\right) \approx 8 \pi \varepsilon \alpha_{P}^{\prime} \ln ^{2}\left(s / s_{0}\right)$, where $R^{2}(s)=B_{a}+B_{b}+\alpha_{P}^{\prime} \ln \left(s / s_{0}\right)$.

This result gives a ground for another method of constructing amplitude. It is possible to consider just from the beginning more complicated singularities of partial amplitudes than usual simple angular momentum poles. It is worth to emphasize that factorization of residues is valid not only for simple $j$-poles but also for any isolated $j$-singularity [2]. It means that one can consider, for instance, double (dipole pomeron)[3] or triple (tripole pomeron) [4] pole instead of simple pole.

\section{Multipomeron exchanges in the model with $\alpha_{P}(0)>1$}

At $s \rightarrow \infty$ the inclusive cross section in central region is dominated by contribution of the diagram on Fig. (1) and can be written in a general form as $E \frac{d^{3} \sigma}{d^{3} p}=g_{a}(0) \mathscr{F}\left(y_{0}-y\right) v_{c}\left(p_{t}^{2}\right) \mathscr{F}\left(y_{0}+\right.$ y) $g_{b}(0)[5,6]$. If the input pomeron is simple $j$-pole and has intercept $\alpha_{P}(0)=1+\varepsilon$ then $\mathscr{F}\left(y_{0} \pm\right.$ $y)=\left(y_{0} \pm y\right)^{2}$. It is necessary to note that this result exactly coincide with those which can be obtained if we assume from the beginning that pomeron at $t=0$ is the triple $j$-pole.

If pomeron contribution to partial amplitude (of elastic scattering) at $t=0$ is proportional to $1 /(j-1)^{v+1}$ then $\mathscr{F}\left(y_{0} \pm y\right)=\left(y_{0} \pm y\right)^{v}, \frac{d n}{d y}(y=0) \propto\left(y_{0}-y\right)^{v}\left(y_{0}+y\right)^{v} \propto \ln ^{v}\left(s / s_{0}\right)$ and $<n>\propto$ $\ln ^{1+v}\left(s / s_{0}\right)$. We would like to remark that such a behaviour of $d n / d y$ (at $v>0$ ) is in a qualitative agreement with high energy experimental data, which show a rise $d n / d y$ at $y_{0}$ and a parabolic form.

\section{Comparison of the unitarized pomeron models with the data}

\subsection{Experimental data}

We consider the data on $E d^{3} \sigma / d^{3} p$ at $\sqrt{s}=200,540,630,900,1800 \mathrm{GeV}$ (240 points) [7, 8] and on $d n / d \eta$ [7, 9] normalized to $\sigma_{\text {in }}$ (48 points).

Even for the chosen high energies we see a nontrivial dependence of cross sections on $p_{t}$. One can clearly see that slope is changing with energy. Taking into account that $p_{t}$-dependence in the pomeron contribution is coming only from vertex function $v_{c}\left(p_{n}^{2}\right)$ one can expect that the slope effect can be explained in the model only due to subasymptotic contributions. For example in the dipole model, they can be simple pole with $\alpha_{P}(0)=1$ as well as $f$-reggeon which is important at not highest energies. Another important feature of the data is a changing an exponential increasing $E d^{3} \sigma / d^{3} p$ at small transverse momenta $p_{t}<1 \mathrm{GeV}$ for a power like one at higher $p_{t}$ (larger than 
$1 \mathrm{Gev})$. These data are given for $y=0$, however another set of data, namely, $d n / d \eta$ is more interesting for our aim. It can be obtained from $E d^{3} \sigma / d^{3} p$ by integration over $p_{t}$ and with a transformation from $y$ to $\eta$.

\subsection{The data fit}

Parameters of the models as well as $\chi^{2}$ obtained in the fits, description of the data is demonstrated on Figs.(2) and (3).

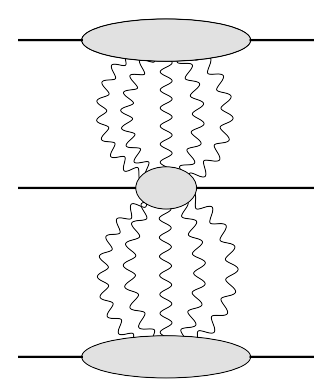

Figure 1: The dominating at $s \rightarrow \infty$ contribution to central inclusive production

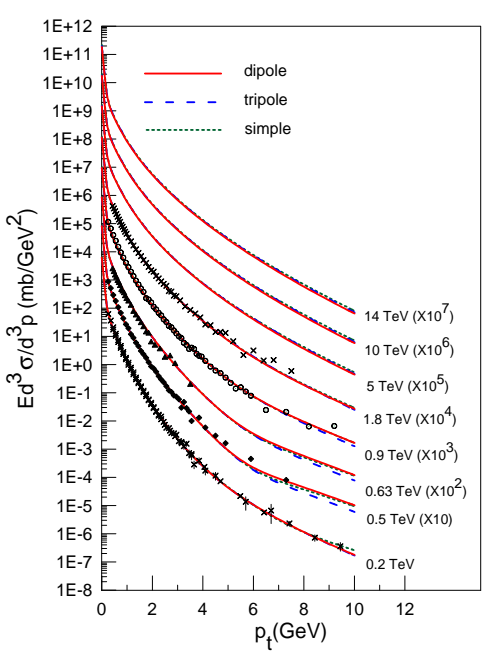

Figure 2: $p_{t}$-dependence of inclusive cross sections at high energies. Data are taken from [7. 8]. Curves correspond to considered models. Red solid line - dipole pomeron model, blue long dashed line - tripole pomeron model, green doted line - simple pomeron pole with $\alpha(0)>1$. Predictions for three LHC energies are also shown.

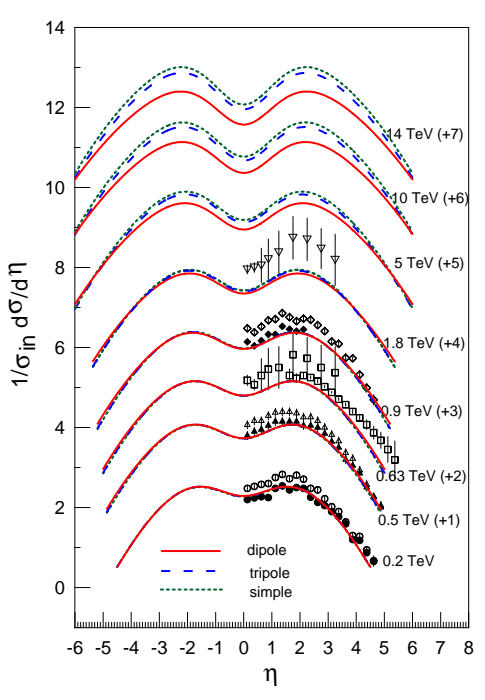

Figure 3: Density of the produced hadrons as function of pseudorapidity and energy. Red (solid), blue (dashed) and green (dotted) lines are theoretical values correspondingly in dipole, tripole and simple pole pomeron model. Solid symbols correspond the data normalized to $\sigma_{i n}$, open symbols correspond to data normalized to $\sigma_{N S D}$ (not used in the fit procedure). Predictions for three LHC energies are also shown.

One can see that theoretical curves in three models are very close each to other, at least for energies where data exist. However a difference between the models' predictions is increasing with energy.

\section{Conclusion}

Thus we have shown that the high energy experimental data on one-particle inclusive distribution can be described well in the models of unitarized pomeron, which do not violate unitarity restrictions. The dipole (tripole) pomeron model, correspondingly lead to $d n / d \eta(y=0) \propto \ln ^{4} s\left(\ln ^{3} s\right)$ and $\left\langle n>\propto \ln ^{3} s\left(\ln ^{2} s\right)\right.$. These models predict a small difference in $d n / d \eta(y=0)$ and $\langle n\rangle$ at low LHC energies which however is increasing with energy. 


\section{References}

[1] S.Donnachie, G.Dosch, P.Lsndshoff, O.Nachtmann, Pomeron Physics and QCD, Cambridge University press, 2002.

[2] J.R. Cudell, E. Martynov, G. Soyez, Nucl. Phys. B682 (2004) 391.

[3] E. Martynov, Phys. Rev. D76 (2007) 074030 and references therein.

[4] M.S. Dubovikov, K.A. Ter-Martirosyan. Nucl.Phys. B124 (1977) 163. See also [3] and refs. therein.

[5] E.S. Martynov, Preprint of Bogolyubov institute for Theoretical physics, ITP-85-26-E, 1985, Kiev.

[6] A.K.Likhoded, O.P.Yushchenko, Int. Journ. Modern Physics A, 6 (1991) 913.

[7] F. Abe, Phys. Rev. Lett. 61 (1988) 1819; F. Abe et al. [CDF Collaboration], Phys. Rev. D41 (1990) 2330; G.J. Alner et al.(UA5 Collaboration), Phys. Rept. 154 (1987) 247; J. Adams et. al., Phys. Rev. Lett. 91 (2003) 172302.

[8] C. Albajar et al., Nucl. Phys. B335 (1990) 261.

[9] G.J. Alner et al.(UA5 Collaboration), ZP C33 (1986) 1; Phys. Rept. 154 (1987) 247. R. Harr et al., Phys. Lett. B401 (1997) 176. 